\title{
Acupuncture Promoted the Recovery of the Neural Plasticity Functions and the Repair of Neural Plasticity-Related Proteins in the Prefrontal Cortex of CUMS-induced Depressed Rats
}

\author{
Wenya Huang \\ Xiamen University https://orcid.org/0000-0003-4696-6874 \\ Peng Li \\ Xiamen University Medical College \\ Wen-jing Cheng \\ Xiamen University Medical College \\ Yang Huang \\ Xiamen University Medical College \\ Wenjie Chen \\ Xiamen University Medical College \\ Yiping Chen \\ Shanxi University of TCM: Shanxi University of Traditional Chinese Medicine \\ Xinnan Wu \\ Xiamen University Medical College \\ Yanxun Huang \\ Xiamen University Medical College \\ Yining Yan \\ Xiamen University Medical College \\ Xianjun Meng ( $\nabla$ mengxianjun@xmu.edu.cn ) \\ Xiamen University Medical College
}

Research

Keywords: Acupuncture therapy, Depression, Neural plasticity, Prefrontal cortex

Posted Date: February 15th, 2021

DOl: https://doi.org/10.21203/rs.3.rs-190556/v1

License: (c) (i) This work is licensed under a Creative Commons Attribution 4.0 International License.

Read Full License 
Page $2 / 23$ 


\section{Abstract}

Background: Acupuncture therapy in Traditional Chinese Medicine has been widely believed as a complementary treatment for depression with numerous clinic and research reports. However, mechanisms of neural plasticity about the positive effects of acupuncture still rarely reported. The purpose of our paper aim to investigate the efficiency of acupuncture in curing depressive-like behaviors and discuss the mechanism underling neural plasticity and its related proteins BDNF/PSD95/SYN/PKMZ in prefrontal cortex.

Methods: 32 rats with the same baseline were divided into 4 groups at random: control group(8rats), model group(8rats), acupuncture group(8rats) and flouxetine group(8rats). Depression rat models were induced by chronic unpredictable mild stress combined with single cage isolation for 6 weeks. Acupuncture group and fluoxetine group then give 3-weeks treatment during the later 3weeks of modeling procedure. The open field test (OFT), elevated-plus-maze (EPM) and sucrose preference test (SPT) were executed to estimate the depressive behaviors. The number of nerve cells, the length of dendrites, and the spines density in prefrontal cortex were observed by Golgi staining. The expression of prefrontal cortex BDNF, PSD95, SYN and PKMZ protein were detected by western blot and their related mRNA were detected by RT-PCR.

Results: Acupuncture could alleviate depressive-like behaviors and promote the recovery of the neural plasticity functions in prefrontal cortex, which exerted similar antidepressant effect as fluoxetine, increasing cells number, prolonging the dendrites length, enhancing the spine density.

Conclusion: Acupuncture therapy could alleviate depressive-like behaviors which may contribute to the recovery of the neural plasticity functions and the repair of neural plasticity-related proteins BDNF/PSD95/SYN/PKMZ in the prefrontal cortex of CUMS-induced depressed rats.

\section{Introduction}

Depression, which is considered to be one of the chronic neurological diseases with continuous symptoms including low self-esteem, helplessness and anhedonia. Patients with severe depression have the highest suicide rates among human being[1]. According to the latest analysis of the World Health Organization's survey, more than 300 million people have suffered from severe depression in the world and with a growth rate of about $18 \%$ in the past decade, thus, depression has become an major cause of human emotional disorders in the world[2, 3]. Studies demonstrated that depression could induce longterm neural plasticity changes in some specific brain regions and the repair of neurogenesis and synaptic plasticity are strongly related to periodontal health and the prognosis of most depressive patients[4-6]. Most antidepressant treatment produces therapeutic action by enhancing neuroplasticity[7]. Acupuncture has been demonstrated to be one of the effective therapies for depression and the pathologies of which are related to neural plasticity $[8,9]$. 
Acupuncture is a vital component of Oriental medicine which widely believed as an effective complementary treatment for depression, with fewer by-effects and lower financial burden. The study about acupuncture promoting neural plasticity has become a research hotspot in the medical community recently $[10,11]$. Researches showed that acupuncture plays an essential role in protecting neurons, which may relieve the symptoms of patients via mediating on neural plasticity[8, 9, 12]. In addition, doctors have achieved significant results in the clinical treatment of depression patients by using acupuncture[13-15]. It is believed that acupuncture is associated with the regulation of cortical striatum reward / motivation circuit in some specific brains of depression patients[16]. Previous study of our research found that acupuncture can reverse the changes of behavior on depression model rats. It can also play a role in treating depression-like behavior by regulating $\beta$-CaMKII in the lateral habenular nucleus of depression model rats in the signaling pathway[17]. But the deep-seated mechanism needs to be further explored.

Prefrontal cortex (PFC) is part of the limbic cortex, which participate in many stress-sensitive psychological disorders because of its rich neural interconnectivity[18, 19]. Researches has demonstrated that neuroplasticity in limbic areas is disrupted in depression, particularly the neural plasticity-related proteins in prefrontal cortex which plays a vital role in pathologies of depression[20-22]. Brain derived neurotrophic factor (BDNF) which is a plasticity-related protein widely distributed in the brain and central nervous system[23, 24]. BDNF plays a critical role in the survival and differentiation of neurons during the development of the brain central nervous system, thus, involving in neurogenesis and neuroplasticity in the brain central nervous system[25, 26]. Postsynaptic density 95 (PSD-95) is a kind of protein connecting structurally to synaptic signaling complexes and receptors [27]. Synaptophysin (SYN) is a marker protein located in the overall glycoprotein of presynaptic nerve development, which is the member of presynaptic vesicle boutons[28, 29]. PSD-95 and SYP are two synapse marker proteins which involved in synaptic signal transmission[30].

PKMZ is short for Protein kinase Mzeta, which is an atypical protein kinase $\mathrm{C}$ subtype in some specific part of the brain. Storing memory and maintaining long-term potentiation are the functions of PKMZ. Studies have shown that Protein kinase Mzeta in prefrontal cortex mediates rats depressive-like behaviors and associated with the morphology of neural dendritic spines[31, 32]. It is currently believed that the damage of neural plasticity-related proteins in some specific part of the brain might associated with the pathologies of depression. The efficacy of acupuncture in the intervention of depression has been widely reported. However, few studies demonstrated the relationship among acupuncture, prefrontal cortex and the neural plasticity-related proteins.

The object of current research was to explore the antidepressant mechanism underlying acupuncture based on the neuroplasticity and its neural plasticity-related proteins BDNF/PSD95/SYN/PKMZ in the prefrontal cortex of CUMS-induced depressed rats. To our best knowledge, it was an unprecedented research to demonstrated the antidepressant effects of acupuncture underling its neural plasticity-related proteins BDNF/PSD95/SYN/PKMZ in prefrontal cortex. 


\section{Materials And Methods}

\subsection{Animals and experimental design}

48 SPF SD male rats weighing 120-150 g, which purchased from the company of Shanghai Slake Laboratory Animal Co., Ltd. with the license number SCXK (Shanghai) 2017-0005. After one week of adaptive feeding, the rats body weight, open field test and sucrose preference test were investigated before the experiment, which ensure that all experimental rats characteristics were in the same baseline. We choose 32 rats with the same baseline of body weight and behavior which met the requirements.

The 32 rats were divided into 4 groups at random: control group $(\mathrm{N}=8)$, CUMS group $(\mathrm{N}=8)$, acupuncture group $(\mathrm{N}=8)$ and fluoxetine group $(\mathrm{N}=8)$. Rats in the control group reared together, while rats in the other three groups were socially isolated by placing each rat in a single cage and underwent CUMS procedure for 6 weeks. Acupuncture group and fluoxetine group then give 3-weeks treatment during the later 3 weeks of modeling procedure (Fig. 1).

\subsection{Establishment of animal model}

According to previous study $[33,34]$, the depression rat model was induced by chronic unpredictable mild stress combined with single cage isolation for 42 days. We chose one stimulus every day and same stimulus could not occur continuously, the modeling procedure and schedule were shown in Table 1. Weight monitoring and behavioral test were conducted to estimate the state of rats. The success criteria of depressive model establishment showed the significant reduction of behavioral score and body weight.

Table 1

CUMS modeling procedure

\begin{tabular}{|c|c|}
\hline stress & day \\
\hline Fasting (24 h) & $1,13 \rrbracket 19 \rrbracket 32 \rrbracket 42$ \\
\hline $45^{\circ} \mathrm{C}$ hot water stimulation( $5 \mathrm{~min}$ ) & $2,10 \otimes 24 \rrbracket 34 \rrbracket 41$ \\
\hline restraint (3 h) & $3,17 \rrbracket 20 \otimes 30 \rrbracket 40$ \\
\hline water deprivation $(24 \mathrm{~h})$ & $4,14 \rrbracket 25 \rrbracket 28 \rrbracket 39$ \\
\hline day and night inversion (24 h) & $5,11 \rrbracket 21 \rrbracket 36 \rrbracket 38$ \\
\hline $4^{\circ} \mathrm{C}$ cold water stimulation $(5 \mathrm{~min})$ & $6,18 \rrbracket 26 \rrbracket 35 \llbracket 37$ \\
\hline squirrel cage tilt $\left(45^{\circ}, 24 \mathrm{~h}\right)$ & $7,15 \rrbracket 22 \rrbracket 33 \rrbracket 36$ \\
\hline noise stimulation ( $3 \mathrm{~h}, 80 \mathrm{~dB})$ & $8,12 \bowtie 27 \llbracket 31$ \\
\hline tail clamping (3 min) & $9,16 \rrbracket 23 \rrbracket 29$ \\
\hline
\end{tabular}

\subsection{Acupuncture and fluoxetine intervention}


As shown in Fig. 2A, rats in acupuncture group were given acupuncture treatment at Shangxing (DU23) and Daling (PC7) before daily CUMS stimulation. Acupuncture operation: Flat needling Shangxing(DU23) toward the direction of the nose, the needle was inserted as deep as $3-5 \mathrm{~mm}$; Obliquely stab Daling(PC7) toward the direction of Neiguan(PC6), the needle was inserted as deep as $2-3 \mathrm{~mm}$. The needles used were sterilized disposable stainless steel needles (0.18 mm diameter; Hanyi, Beijing, China). As shown in Fig. 2B, rats in fluoxetine group were given fluoxetine (PHR1394-1G, Sigma-aldrich) solution which $(0.21 \mathrm{mg} / \mathrm{ml})$ by gavage $(1 \mathrm{ml} / 100 \mathrm{~g})$ before daily stimulation.

\subsection{Body weights and general status.}

Rats body weights were monitored once a week, observe the rat's mental state, eating status, activity level and hair gloss every day.

\subsection{Behavioral tests}

Behavioral tests including open field test, elevated-plus-maze and sucrose preference test were executed at the end of experiment.

\subsubsection{Open field test}

OPT was executed to test the locomotor activity and depressive-like behaviors of rats, the operational steps is conducted as previous study[35]. We put the rats in the test environment for adaption prior to the experiment. The open field region consists of the black acrylic device $(100 \mathrm{~cm}$ in length and width, $40 \mathrm{~cm}$ in height). When the test started, rats were first placed in the center of area, then allowed to travel freely during the whole area. We observed the autonomic activity of rats during the 5 minutes test (recorded by video camera and analyzed by SMART software). The change of total crossing distance and central residence time were assessed as the criteria of depressive symptom. After every test, $75 \%$ ethanol was used to disinfect the experimental site.

\subsubsection{Elevated-plus-maze test}

Elevated-plus-maze test was used to estimate the anxiety-like behavior of depressive rats as reported in previous studies[36, 37]. The elevated plus-maze devices have one central zone $(10 \times 10 \mathrm{~cm})$ together with two open arms $(50 \times 10 \times 40 \mathrm{~cm})$ and two closed arms $(50 \times 10 \times 40 \mathrm{~cm})$, the arm specifications is $50 \mathrm{~cm}$ in length, $10 \mathrm{~cm}$ in width and $40 \mathrm{~cm}$ in height. When the test started, rats were first placed in the center of area and their heads facing the open arms. We observed the autonomic activity of rats for crossing the open arms and the closed arms during the 5 minutes test (recorded by video camera and analyzed by SMART software). After every test, $75 \%$ ethanol was used to disinfect the experimental site.

\subsubsection{Sucrose preference test}

Sucrose preference test was executed to examine the anhedonia of rats, the operational steps is conducted as previous study[38]. We trained rats to adapt to sucrose-water 2 days before the real test begun: each cage was equipped with one $1 \%$ sucrose bottle and one pure water bottle, we exchanged the 
position of two bottles every 12 hours. After the adaptive phase, water and food prohibition in rats for 24 hours. Then start the formal test: each rat was given two bottles (one $1 \%$ sucrose and one pure water) which had been quantified beforehand, we removed bottles after 12 hours and calculated the liquid consumption. Sucrose preference $(\%)=$ [sucrose solution consumption / (sucrose solution consumption + pure water consumption)] $\times 100$.

\subsection{Extraction and detection}

Fasting and water deprivation for 12 hours before the extraction procedure. After animals were anesthetized with $25 \%$ pentobarbital sodium $(50 \mathrm{mg} / \mathrm{kg}$, intraperitoneal injection), we used cervical dislocation to prevent pre-synaptic and post-synaptic effects of anesthesia. Abdominal aorta blood collection and brain removal after euthanasia, then the prefrontal cortex harvested rapidly under ice. The number of nerve cells, the length of dendrites, and the density of dendritic spines were observed by Golgi staining. The expression of prefrontal cortex BDNF, PSD95, SYN and PKMZ protein were detected by western blot and their related mRNA were detected by RT-PCR.

\subsubsection{Golgi Staining and Evaluation of Dendritic Spine Density}

After the rat euthanasia, immediately take the prefrontal cortex and fix it in the fixative for more than $48 \mathrm{~h}$. Cut the prefrontal cortex tissue into tissue blocks with thickness of 2-3mm according to the tissue site to be observed, gently rinse the brain tissue with normal saline for several times, then place in a $45 \mathrm{ml}$ round bottom EP tube, add Golgi-cox staining solution to completely submerge the brain tissue, and place in a cool and ventilated place and avoid light to treat for 14 days (after $48 \mathrm{~h}$ soak, change the new staining solution, and then change the new staining solution every 3 days, total of 14 days). Immerse in distilled water for 3 times, pour in $80 \%$ glacial acetic acid to immerse the tissue overnight, wait for the tissue to become soft, then wash with distilled water, and place into 30\% sucrose. Cut the tissue into 100 microns with oscillating microtome, paste it on a gelatin slide, and dry in the dark overnight. Treat dried tissue slides with concentrated ammonia water for 15 minutes, wash with distilled water for 1 minute, treat with acid hardening fixing solution for 15 minutes, wash with distilled water for 3 minutes, dry and seal the section with glycerin gelatin. Microscope inspection, image acquisition and analysis, obtain panoramic images of brain tissue by panoramic multi-layer scanning with digital slice scanner. Analyses were performed according to standard protocols of our laboratory [39]. The number of spinous cells was observed in the pyramidal neuron dendrites of the prefrontal cortex. We used Sholl analysis method for dendritic tree examination[40]. The density of the spine was quantified and the number of spines along the length was calculated[41].

\subsubsection{Western blot}

The contents of BDNF, PSD95, SYN and PKMZ in prefrontal cortex were determined by Western-blot. The methods was described previously[42]: We took $30 \mu \mathrm{g}$ samples from brain tissue and added with 4xloading buffer, put them in boiling water for $10 \mathrm{~min}, 10000 \mathrm{rpm}$ centrifugation for $10 \mathrm{~min}$; SDS-PAGE 
gel with $10 \%$ or $12 \%$ according to protein molecular mass was prepared for electrophoretic separation of protein samples; SDS-PAGE gel protein was transferred to PVDF membrane by electrophoresis; PVDF was immersed in sealed solution containing 5\% skimmed milk powder after electrophoresis, incubated for 60 minutes during room temperature condition; PBST washed membrane was incubated at $3 \times 5 \mathrm{~min}$. Add the corresponding antibody, stay at 4 Celsius condition overnight, then wash PBST for 3 times, 20 min each time, add the corresponding HRP labeled two anti-solution, incubated for 60 minutes during room temperature condition, PBST wash 3 times, 10 min each time. The PVDF film was reacted with the freshly prepared enhanced chemiluminescence agent (ECL) solution to $2 \mathrm{~min}$, and was rapidly exposed and developed in the darkroom. The gray value of the target protein was analyzed by the gel image system.

\subsubsection{Reverse transcription-polymerase chain reaction (RT- PCR).}

We searched the sequences of BDNF, PSD95, SYN and PKMZ mRNA codes from the consensus coding sequence database of the National Biotechnology Information Center ((NCBI)). The detailed primer sequences for BDNF, PSD95, SYN, PKMZ and the beta-actin control are shown in Table 2. PCR primers were developed before the RT-PCR operation.

Table 2

PCR Primer Information Sheet

\begin{tabular}{|lll|}
\hline mRNA Primers & Sequence(5'-3') & Product leng(bp) \\
\hline Rat- $\beta$-actin-F & CTGGCTCCTAGCACCATGAA AAAACGCAGCTCAGTAACAGTC & 180 \\
Rat- $\beta$-actin -R & GCCTCCTCTGCTCTTTCT & 141 \\
Rat- $\beta$-BDNF-F & GCCGTTACCCACTCACTA & 142 \\
Rat- $\beta$-BDNF-R & ACACCCATTGCCCAGAAC & 209 \\
Rat- $\beta$-PSD95-F & TCTCCACGCAGTCATAAAG & 166 \\
Rat- $\beta$-PSD95-R & ACAGCAGTGTTCGCTTTCA & \\
Rat- $\beta-S Y P-F$ & CAGAGCACCAGGTTCAGG & \\
Rat- $\beta-S Y P-R$ & CACATTAAGCTGACGGACTA & \\
Rat- $\beta-P K M Z-F$ & TCTCAAACATAAGGACACCC & \\
Rat- $\beta-P K M Z-R$ & & \\
\hline
\end{tabular}

The tissue samples of about $100 \mathrm{mg}$ were weighed, grinded with liquid nitrogen, cracked with $1 \mathrm{ml}$ trizol at room temperature for $5 \mathrm{~min}$, centrifuged at $4{ }^{\circ} \mathrm{C}$ for $10 \mathrm{~min}$ with $12000 \mathrm{rpm}$, and the supernatant was obtained. $0.2 \mathrm{ml}$ chloroform was added to each $1 \mathrm{ml}$ cleavage solution and incubated for 3 minutes during room temperature condition, then centrifuged $10 \mathrm{~min}$ at $4{ }^{\circ} \mathrm{C} 12000 \mathrm{rpm}$. The sample is divided into three layers, the top layer is taken, $500 \mu \mathrm{L}$ aqueous phase is absorbed and $250 \mu \mathrm{L}$ anhydrous ethyl alcohol is added, then the mixture is transferred to the adsorption column and centrifuged by $12000 \mathrm{rpm}$ for $45 \mathrm{~s}$. 
Add 500 ul deproteinized solution 12000rpm to centrifuge for $45 \mathrm{~s}$, add $500 \mathrm{ul}$ rinse solution to rinse twice, $12000 \mathrm{rpm}$ centrifuge for $45 \mathrm{~s}$, elute RNA and determine the concentration to run glue identification. 1ug was extracted from each sample for reverse transcription, and each sample was detected by BDNF, PSD95, SYN, PKMZ and the beta-actin primers respectively. Finally, $2 \mu \mathrm{L}$ PCR products were detected by agarose gel. The electrophoresis conditions were $140 \mathrm{~V}, 15 \mathrm{~min}$, gel imaging. We analyzed the gel using ImageJ software (National Institutes of Health, Bethesda, Maryland).

\subsection{Statistical analysis}

All data were processed by SPSS 21.0 software. The statistical data of each test index are expressed by means of mean and standard deviation $(x \pm s)$. Firstly, the normality and square deviation are tested. If the variance is homogeneous, LSD method is selected; if the variance is uneven, games Howell method is selected for variance analysis; if not, rank sum test is selected. The difference was statistically significant $(p<0.05)$.

\section{Results}

\subsection{Effects of CUMS and intervention treatments on body weight.}

As shown in Fig. 3B, before the experiment, there is no differences in rats body weight baseline among all groups $(p>0.05)$; while with the progress of the experiment, the weight gain trend of the CUMS- model rats all showed lower levels comparing with the control rats $(p<0.001)$. As we can see in Fig. 3C, after the experiment, CUMS rats showed a significant reduction in body weight $(\mathrm{p}<0.001)$ comparing with the control group; Rats in acupuncture group showed a significant growth in body weight $(p<0.001)$ comparing with the CUMS group; while there is no significant differences in rats body weight between CUMS and Fluoxetine group $(p>0.05)$.

\subsection{Effects of CUMS and intervention treatments on behavior tests.}

As shown in Fig. 4A, the results of open-field test showed a significant reduction in total crossing distance of model rats $(p<0.01)$ comparing to the control rats; After the intervention treatments, acupuncture group and the fluoxetine group all showed an increase trend of total distance comparing to the model group, but there are not significant differences $(p>0.05)$. As shown in Fig. $4 B$, the results of the elevated plus maze showed a significant reduced time in open arms in the model group comparing to the control group $(\mathrm{p}<$ $0.001)$; the reduced time in open arms was significantly increased in the acupuncture group $(p<0.05)$ comparing to the model group, but the fluoxetine group showed no statistical significance $(p>0.05)$. As we can see from the results of sucrose preference test(Fig. 4C), the model group showed a significant decreased in the $1 \%$ sucrose intake comparing to the control group $(p<0.01)$; After the intervention treatments, the intake of $1 \%$ sucrose was significantly increased in the acupuncture group and the fluoxetine group $(p<0.01, p<0.01)$ comparing to the model group. 


\subsection{Effects of CUMS and intervention treatments on Golgi staining.}

As shown in Fig. 5, Golgi staining of rat prefrontal cortex, the arrangement of cells distribute in the control rats were more neatly, and showed a clearer outline than CUMS group. The cells in CUMS group were disorderly arranged with unclear outline, and the number of dendrites is decreased, the length of dendrites is shorter. After the intervention treatments, the acupuncture group and the fluoxetine group all increased cells number, prolonged the dendrites length, enhanced the spine density comparing with the CUMS model group.

\subsection{Effects of CUMS and intervention treatments on morphological character of dendritic arborization and spine density of prefrontal cortex.}

As shown in Fig. 6, the dendritic arborization were rich and well developed in control group A; After the CUMS procedure, the development dendritic spine impaired in the CUMS group (Fig. 6B); After the intervention treatments, acupuncture and fluoxetine are able to restore the impaired dendritic spines, with enlarged spiny dendritic branches in the acupuncture group(Fig. 6C)and the fluoxetine group(Fig. 6D) comparing with the CUMS model group. As shown in Fig. 6E, the CUMS model group showed lower level of intersection per shell of pyramidal neuron arborization comparing with control group; After the intervention treatments, acupuncture group and the fluoxetine group all increase the level of intersection per shell. As shown in Fig. 6F, the sum of intersections of dendrites in the CUMS group showed a significant decrease $(p<0.01)$ comparing to the control group; After the intervention treatments, the sum of intersections of dendrites in acupuncture group and the fluoxetine group all showed significant increases $(p<0.01, p<0.05)$. As shown in Fig. 6G, the CUMS model group showed lower level of density of dendritic spines comparing with control group, but no statistical significance $(p>0.05)$; After the intervention treatments, the fluoxetine group showed a significant increase of the dendritic spine density comparing with the CUMS model group $(p<0.05)$, while the acupuncture group showed no statistical increase $(p>0.05)$.

\subsection{Effects of CUMS and intervention treatments on the protein expression of BDNF, PSD95, SYN and PKMZ in prefrontal cortex measured by western-blot.}

As shown in Fig. 7, the protein expression of BDNF, PSD95, SYN and PKMZ in prefrontal cortex of the CUMS model group decreased $(p<0.05, p<0.01, p<0.01, p<0.01)$ comparing to the control group; After the intervention treatments, the protein expression of BDNF, PSD95, SYN and PKMZ in prefrontal cortex of 

0.05).

\subsection{Effects of CUMS and intervention treatments on the mRNA level of BDNF, PSD95, SYN and PKMZ in prefrontal cortex measured by RT-PCR.}

As shown in Fig. 8, the mRNA level of BDNF, PSD95, SYN and PKMZ in prefrontal cortex of the CUMS model group all decreased $(p<0.05, p<0.01, p<0.01, p<0.001)$ comparing to the control group; After the intervention treatments, the mRNA level of PSD95, SYN and PKMZ in prefrontal cortex of the acupuncture group and the fluoxetine group all increased compared with the CUMS model group, showing significant differences $(p<0.05$ or $p<0.01)$.

\section{Discussion}

In our current research, the CUMS model was established successfully according to the results of behavior test. Under the 6-weeks chronic unpredictable mild stress, rats showed less sucrose consumption in SPT, less total distances crossing in OPT as well as less open arms during EPM than rats in control groups. And their body weight grew slowly that the control rats. It is gratifying to find that those depressive-like behavior can be ameliorated by prolonged acupuncture treatment and fluoxetine intervention. These consequences indicated that the acupuncture and fluoxetine are effective to treat the CUMS induced depressive rats, thus showing the antidepressant-effect as proved in previous study $[43,44]$.

At present, antidepressant drugs are still in the leading position in clinic[45], fluoxetine in particularly; however, most antidepressant drugs have slow onset and low efficacy with severe side effects[46]. Acupuncture, as a safe and effective alternative therapy for depression with fewer side effects. The choice of acupoints is a key factor to ensure the curative effects of acupuncture. Daling (PC7) is considered to be the original acupoint of pericardium meridian. According to the meridian-collateral theory in Traditional Chinese Medicine, Daling (PC7) can not only relieve the fire evil enveloped by the heart, but also has great efficacy in treating mental diseases which can calm the mind and benefit the brain nerves. Shangxing (DU23) located in the governor meridian, and is good at supervising and awakening the mind. These two points are especially effective for mental illness. Therefore, the acupoints of Daling and Shangxing were elected in the acupuncture treatment group, and fluoxetine was used as intervention control group because it is one of the most commonly used antidepressants in clinic. To further explore the deep-seated mechanism of antidepressant-effect of acupuncture and fluoxetine, the Golgi staining and evaluation of dendritic spine density were used. Then, Western blot (WB) and Reverse transcription-polymerase chain reaction (RT-PCR) were be used to detect the neural plasticity-related proteins BDNF/PSD95/SYN/PKMZ and their mRNA level. 
Neural plasticity, also known as brain plasticity, containing structural plasticity, functional plasticity and the cellular and molecular mechanisms associated with them[47]. Literature research indicated that the biological mechanism of depression associate with the neural plasticity in specific brain areas, especially the prefrontal cortex[48]. The number and function of synapses in central nervous system of depression were obviously disordered[49]. As a basic mechanism of neuronal adaptation, neural plasticity is destroyed in animal models of emotional disorder and stress. Chronic stress can promote or aggravate depression and destroy neural plasticity, while antidepressant treatment has the opposite effect and can enhance neural plasticity[50]. Our current results of Golgi staining showed that the arrangement of cells distributed in the CUMS rat prefrontal cortex was disorderly arranged with unclear outline, and the number of dendrites is decreased, the length of dendrites is shorter. Morphological analyses of dendritic arborization and spine density of CUMS rat prefrontal cortex also impaired. After the intervention treatments, acupuncture and fluoxetine all increased cells number, prolonged the dendrites length, enhanced the spine density comparing with the CUMS model group. Moreover, acupuncture and fluoxetine are able to restore the impaired dendritic spines, with enlarged spiny dendritic branches, increasing level of intersection per shell and intersections of dendrites comparing with the CUMS model group. Our results are consistent with the report: CUMS can promote or aggravate depression and destroy neural plasticity, while acupuncture and fluoxetine treatment have the opposite effect and can enhance neural plasticity.

Synaptic formation and neural plasticity are regulated by complex molecular protein interactions. The destruction of many key proteins is related to the susceptibility to depression, including brain derived neurotrophic factor (BDNF), post synaptic density 95(PSD-95), synaptophysin (SYN) and protein kinase Mzeta (PKMZ). We choose those proteins as they were proved to be the neural plasticity-related proteins. BDNF can regulate the neural plasticity and synaptic transmission by increasing $\mathrm{Ca} 2+$ levels and mitochondrial movement in neurons. The transportation and distribution of mitochondria play a vital role in BDNF-mediated synaptic transmission. Research show that BDNF enhances the release of neurotransmitter to protect neuroplasticity[51]. Endogenous BDNF is effective for cannabinoid-mediated neurogenesis[52]. As a scaffold of synaptic components required for synapse development, PSD-95 is the main organizer of signal complexes on the postsynaptic membrane. Impaired synaptic function can lead to neuropsychiatric diseases, collectively referred to as synaptic diseases. The genetic decrease in PSD95 protein levels may affect postsynaptic function and plasticity[53]. The synaptophysin mutant synapse is a weak donor at the presynaptic terminal. Previous research indicated that synaptophysin can regulate the formation of activity-dependent synapses. Previous studies have shown that the decreased levels of neuronal synapses can change neuronal circuits and the process might be associated with the mechanism of mental diseases[54]. Protein kinase Mzeta (PKMZ) is a constitutively active N-terminal truncated form of PKC- $\zeta$, has long been implicated in a cellular correlate of learning, long-term potentiation (LTP). The regulation of PKM- $\zeta$ in the brain can affect cerebellar-dependent learning and memory[55]. Plasticity-related protein kinase Mzeta can promote neurite shaft protrusion which is closely related to mental illness. 
In our current results, the protein expression and mRNA level of BDNF, PSD95, SYN and PKMZ in prefrontal cortex of the CUMS model group decreased, After the intervention treatments, the protein expression and mRNA level of BDNF, PSD95, SYN and PKMZ in prefrontal cortex of the acupuncture group and the fluoxetine group all increased comparing to the CUMS model group. Those results are consistent with previous assumption that acupuncture promote the repair of neural plasticity-related proteins BDNF/PSD95/SYN/PKMZ in the prefrontal cortex of CUMS-induced depressed rats.

\section{Conclusions}

In conclusion, the current research suggests that acupuncture is effective to treat the CUMS induced depressive rats, which could ameliorate the depression-like behavior, increase cells number, prolong the dendrites length and enhance the spine density. The recovery of the neural plasticity functions by enlarging spiny dendritic branches, increasing the level of intersection per shell and intersections of dendrites. These alterations may contribute to the repair of neural plasticity-related proteins BDNF/PSD95/SYN/PKMZ in prefrontal cortex. The present study provides new insights into the antidepressant effects of acupuncture underling neural plasticity and its related proteins. However, there are some limitations need to be improved: more brain region should be considered but not just the prefrontal cortex; Different depressed subject has individual difference so it is not reasonable to put them together as a whole. In future researches, we will try to put more samples and choose different brain regions so as to provide comprehensive evidences for the acupuncture treatment in depression.

\section{Abbreviations}

CUMS: Chronic unpredictable mild stress; AP: Acupuncture; FX: Fluoxetine; OFT: The open field test; EPM: elevated-plus-maze; SPT: Sucrose preference test; WB: Western blot; RT-PCR: Reverse transcriptionpolymerase chain reaction; BDNF: Brain derived neurotrophic factor; PSD95: Postsynaptic density 95; SYN: Synaptophysin; PKMZ: Protein kinase Mzeta; PFC: Prefrontal cortex.

\section{Declarations}

\section{Acknowledgments}

The department of Traditional Chinese Medicine in Medical College of Xiamen University is thanked for the invaluable contribution to the study. The authors would also like to thank the participants for volunteering their time to the study.

\section{Funding}

This study was supported by the Natural Science Foundation of Fujian Province of China (NO.2018J01135). 


\section{Data Availability}

The original data (including MNOVA, Excel, and USP format) used to support the fndings of this study were supplied by Xianjun Meng under license and so cannot be made freely available. Requests for access to these data should be made to Xianjun Meng, mengxianjun@xmu.edu.cn.

\section{Author's contributions}

Xianjun Meng was responsible for the study concept and design. Wenya Huang and Peng Li conducted animal experiment and wrote the manuscript. Wenjing Chen, Yang Huang and Wenjie Chen conducted the experiments and collected the data. Yiping ChenखXinnan Wu, Yanxun Huang and Yining Yan helped to analyze the data. All authors approved the final version of the manuscript.

\section{Conflict of interest}

The authors declare that they have no conflicts of interest.

\section{Consent for publication}

Not applicable. The manuscript does not include details, images, or videos relating to individual participants.

\section{Ethics approval and consent to participate}

In this study, all rat care and experimental procedures were approved by the Animal Care and Use Committee of Xiamen University (License No. SCXK 2014-0001) and implemented in accordance with the National Institutes of Health Guidelines for the Nursing and Use of Laboratory Animals.

\section{Author details}

${ }^{1}$ Department of Traditional Chinese Medicine in Medical College, Xiamen University, Xiamen 361005, China.

2 Third Clinical College『Shanxi University of Traditional Chinese Medicine『Shanxi 030619®China.

${ }^{3}$ School of life sciences, Xiamen University, Xiamen 361005, China.

\section{References}


1. Incze MA. I'm Worried About Depression-What Should I Know? JAMA Intern Med, 2019.

2. Global regional. and national incidence, prevalence, and years lived with disability for 354 diseases and injuries for 195 countries and territories, 1990-2017: a systematic analysis for the Global Burden of Disease Study 2017. Lancet. 2018;392(10159):1789-858.

3. Barnett R. Depression Lancet. 2019;393(10186):2113.

4. Singhal G, Baune BT. Microglia: An Interface between the Loss of Neuroplasticity and Depression. Front Cell Neurosci. 2017;11:270.

5. Doan L, Manders T, Wang J, Neuroplasticity underlying the comorbidity of pain and depression. Neural Plast, 2015. 2015: p. 504691.

6. Calabro M, et al. Neuroplasticity, Neurotransmission and Brain-Related Genes in Major Depression and Bipolar Disorder: Focus on Treatment Outcomes in an Asiatic Sample. Adv Ther. 2018;35(10):1656-70.

7. Pittenger C, Duman RS. Stress, depression, and neuroplasticity: a convergence of mechanisms. Neuropsychopharmacology. 2008;33(1):88-109.

8. Liu CZ, Kong J, Wang K, Acupuncture Therapies and Neuroplasticity. Neural Plast, 2017. 2017: p. 6178505.

9. Liu CZ, Chen JD, Zhang M, Advances on the Acupuncture Therapies and Neuroplasticity. Evid Based Complement Alternat Med, 2018. 2018: p. 7231378.

10. Cao J, et al. Analgesic Effects Evoked by Real and Imagined Acupuncture: A Neuroimaging Study. CEREBRAL CORTEX. 2019;29(8):3220-31.

11. Garland SN, et al. Acupuncture Versus Cognitive Behavioral Therapy for Insomnia in Cancer Survivors: A Randomized Clinical Trial. J Natl Cancer Inst. 2019;111(12):1323-31.

12. Tao J, et al. Electro-acupuncture at LI11 and ST36 acupoints exerts neuroprotective effects via reactive astrocyte proliferation after ischemia and reperfusion injury in rats. BRAIN RESEARCH BULLETIN. 2016;120:14-24.

13. Armour M, et al., Acupuncture for Depression: A Systematic Review and Meta-Analysis. JOURNAL OF CLINICAL MEDICINE, 2019. 8(11408).

14. Chan $Y$, et al. The benefit of combined acupuncture and antidepressant medication for depression: $A$ systematic review and meta-analysis. JOURNAL OF AFFECTIVE DISORDERS. 2015;176:106-17.

15. MacPherson $\mathrm{H}$, et al., Acupuncture and Counselling for Depression in Primary Care: A Randomised Controlled Trial. PLOS MEDICINE, 2013. 10(e10015189).

16. Wang $Z$, et al. Acupuncture treatment modulates the corticostriatal reward circuitry in major depressive disorder (vol 84, pg 18, 2017). JOURNAL OF PSYCHIATRIC RESEARCH. 2017;87:105-5.

17. Huang W, et al. Nitric Oxide and Cyclic Guanosine Monophosphate Signaling Mediates the Antidepressant Effects of Acupuncture in the Rat Model of Chronic Unpredictable Mild Stress. Med Sci Monit. 2019;25:9112-22. 
18. Hultman R, et al. Dysregulation of Prefrontal Cortex-Mediated Slow-Evolving Limbic Dynamics Drives Stress-Induced Emotional Pathology. Neuron. 2016;91(2):439-52.

19. Wood M, et al. Infralimbic prefrontal cortex structural and functional connectivity with the limbic forebrain: a combined viral genetic and optogenetic analysis. Brain Struct Funct. 2019;224(1):73-97.

20. David J, et al. L-alpha-amino adipic acid provokes depression-like behaviour and a stress related increase in dendritic spine density in the pre-limbic cortex and hippocampus in rodents. Behav Brain Res. 2019;362:90-102.

21. Pirnia T, et al. Electroconvulsive therapy and structural neuroplasticity in neocortical, limbic and paralimbic cortex. Transl Psychiatry. 2016;6(6):e832.

22. Sairanen M, et al. Chronic antidepressant treatment selectively increases expression of plasticityrelated proteins in the hippocampus and medial prefrontal cortex of the rat. Neuroscience. 2007;144(1):368-74.

23. Kowianski P, et al. BDNF: A Key Factor with Multipotent Impact on Brain Signaling and Synaptic Plasticity. Cell Mol Neurobiol. 2018;38(3):579-93.

24. Leal G, Comprido D, Duarte CB. BDNF-induced local protein synthesis and synaptic plasticity. Neuropharmacology, 2014. 76 Pt C: p. $639-56$.

25. Wang B, et al., Low-Intensity Extracorporeal Shock Wave Therapy Enhances Brain-Derived Neurotrophic Factor Expression through PERK/ATF4 Signaling Pathway. Int J Mol Sci, 2017. 18(2).

26. Lu B, Nagappan G, Lu Y. BDNF and synaptic plasticity, cognitive function, and dysfunction. Handb Exp Pharmacol. 2014;220:223-50.

27. Szogi T, et al., Effects of the Pentapeptide P33 on Memory and Synaptic Plasticity in APP/PS1 Transgenic Mice: A Novel Mechanism Presenting the Protein Fe65 as a Target. Int J Mol Sci, 2019. 20(12).

28. Inoue $Y$, et al. Homer1a regulates the activity-induced remodeling of synaptic structures in cultured hippocampal neurons. Neuroscience. 2007;150(4):841-52.

29. Tarsa L, Goda Y. Synaptophysin regulates activity-dependent synapse formation in cultured hippocampal neurons. Proc Natl Acad Sci U S A. 2002;99(2):1012-6.

30. Han $M$, et al. UV irradiation to mouse skin decreases hippocampal neurogenesis and synaptic protein expression via HPA axis activation. Sci Rep. 2017;7(1):15574.

31. Yan W, et al., Correction: Protein kinase Mzeta in medial prefrontal cortex mediates depressive-like behavior and antidepressant response. Mol Psychiatry, 2019.

32. Yan W, et al. Protein kinase Mzeta in medial prefrontal cortex mediates depressive-like behavior and antidepressant response. Mol Psychiatry. 2018;23(9):1878-91.

33. Lu J, et al. Potential antiinflammatory effects of acupuncture in a chronic stress model of depression in rats. Neurosci Lett. 2016;618:31-8.

34. Wang Q, et al. The recent progress in animal models of depression. Prog Neuropsychopharmacol Biol Psychiatry. 2017;77:99-109. 
35. Kraeuter AK, Guest PC, Sarnyai Z, The Open Field Test for Measuring Locomotor Activity and AnxietyLike Behavior. Methods Mol Biol, 2019. 1916: p. 99-103.

36. Walia V, Garg C, Garg M. NO-sGC-cGMP signaling influence the anxiolytic like effect of lithium in mice in light and dark box and elevated plus maze. Brain Res. 2019;1704:114-26.

37. Kraeuter AK, Guest PC, Sarnyai Z, The Elevated Plus Maze Test for Measuring Anxiety-Like Behavior in Rodents. Methods Mol Biol, 2019. 1916: p. 69-74.

38. Liu MY, et al. Sucrose preference test for measurement of stress-induced anhedonia in mice. Nat Protoc. 2018;13(7):1686-98.

39. Garcia-Rojo G, et al. The ROCK Inhibitor Fasudil Prevents Chronic Restraint Stress-Induced Depressive-Like Behaviors and Dendritic Spine Loss in Rat Hippocampus. Int J Neuropsychopharmacol. 2017;20(4):336-45.

40. Gibb R, Kolb B. A method for vibratome sectioning of Golgi-Cox stained whole rat brain. J Neurosci Methods. 1998;79(1):1-4.

41. Ferreira TA, et al. Neuronal morphometry directly from bitmap images. Nat Methods. 2014;11(10):982-4.

42. Park $\mathrm{H}$, et al. Acupuncture stimulation at $\mathrm{HT7}$ alleviates depression-induced behavioral changes via regulation of the serotonin system in the prefrontal cortex of maternally-separated rat pups. $J$ Physiol Sci. 2012;62(4):351-7.

43. Wang Y, et al., Antidepressant Mechanism Research of Acupuncture: Insights from a Genome-Wide Transcriptome Analysis of Frontal Cortex in Rats with Chronic Restraint Stress. Evid Based Complement Alternat Med, 2017. 2017: p. 1676808.

44. Wang Y, et al. Genome-wide transcriptome analysis of hippocampus in rats indicated that TLR/NLR signaling pathway was involved in the pathogenisis of depressive disorder induced by chronic restraint stress. Brain Res Bull. 2017;134:195-204.

45. Berton O, Nestler EJ. New approaches to antidepressant drug discovery: beyond monoamines. NATURE REVIEWS NEUROSCIENCE. 2006;7(2):137-51.

46. Khin NA, Exploratory Analyses of Efficacy Data From Major Depressive Disorder Trials Submitted to the US Food and Drug Administration in Support of New Drug Applications (vol 72, pg 464, 2011). JOURNAL OF CLINICAL PSYCHIATRY, 2011. 72(6): p. 874-874.

47. Cocks G, et al., Neural Plasticity and Neurogenesis in Mental Disorders. Neural Plasticity, 2016. 2016: p. 1-2.

48. Jensen M, Overgaard M. Neural Plasticity and Consciousness. Frontiers in Psychology, 2011. 2.

49. Hall FS, Li A, Li B, Neural Plasticity in Mood Disorders. Neural Plasticity, 2018. 2018: p. 1-2.

50. Yu H, et al. Inhibition of Phosphodiesterase 4 by FCPR03 Alleviates Lipopolysaccharide-Induced Depressive-Like Behaviors in Mice: Involvement of p38 and JNK Signaling Pathways. Int J Mol Sci. 2018;19(2):513. 
51. Brain-derived Neurotrophic Factor Controls Mitochondrial Transport in Neurons. J Biol Chem. 2014;289(3):1227-7.

52. Ferreira FF, et al., Brain-Derived Neurotrophic Factor (BDNF) Role in Cannabinoid-Mediated Neurogenesis. Frontiers in Cellular Neuroscience, 2018. 12.

53. Fossati G, et al. Reduced SNAP-25 increases PSD-95 mobility and impairs spine morphogenesis. Cell death differentiation. 2015;22(9):1425-36.

54. Tarsa L, Goda Y, Synaptophysin regulates activity-dependent synapse formation in cultured hippocampal neurons. Proceedings of the National Academy of Sciences - PNAS, 2002. 99(2): p. 1012-1016.

55. Chihabi K, Morielli AD, Green JT. Intracerebellar infusion of the protein kinase M zeta (PKMZ) inhibitor zeta-inhibitory peptide (ZIP) disrupts eyeblink classical conditioning. Behav Neurosci. 2016;130(6):563-71.

\section{Figures}

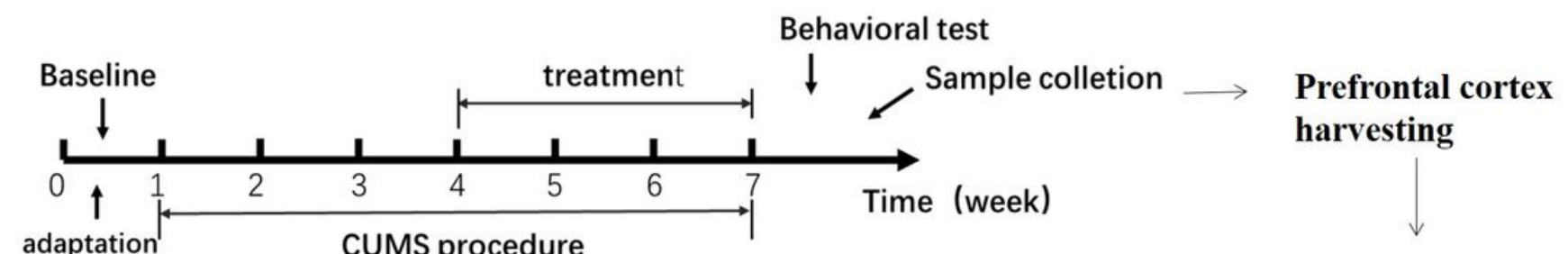

adaptation CUMS procedure

\begin{tabular}{l|c|c|c|c|}
\hline Parameters/groups & Control & Model & AP & FX \\
\hline Social isolation for 6 weeks & - & + & + & + \\
Induction of CUMS for 6 weeks & - & + & + & + \\
Acupuncture at DU23 and PC7 for 3 weeks & - & - & + & - \\
Administered with fluoxetine by gavage for 3 weeks & - & - & - & + \\
\hline
\end{tabular}

The expression of prefrontal cortex BDNF,PSD95 and SYN protein were detected by western blot and their related mRNA were detected by RTPCR.

The number of nerve cells, the length of dendrites, and the density of dendritic spines were observed by Golgi staining.

\section{Figure 1}

Experimental flow chart. 1 week adaptation, 6 week CUMS procedure, 3 week treatment, behavioral test and prefrontal cortex harvesting at the end of experiment. 


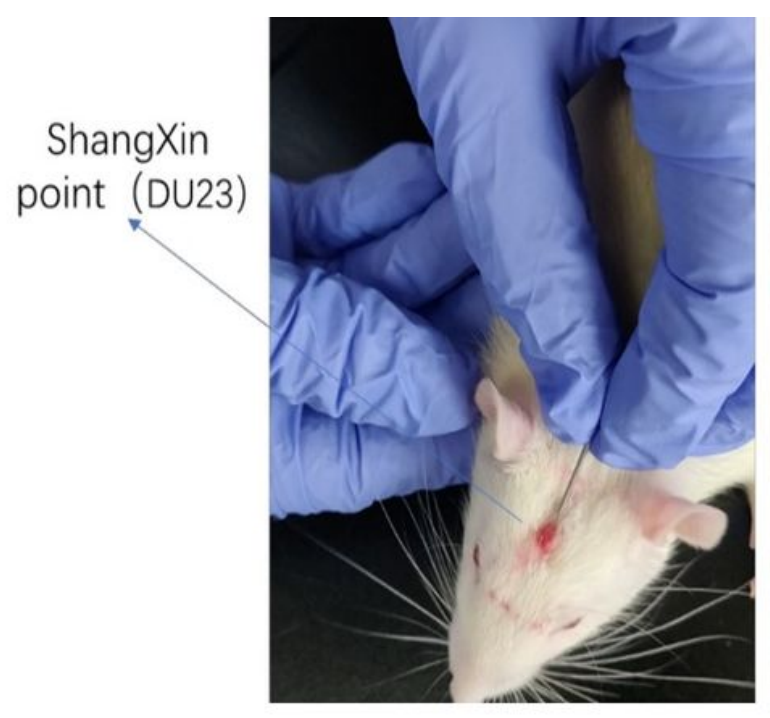

A

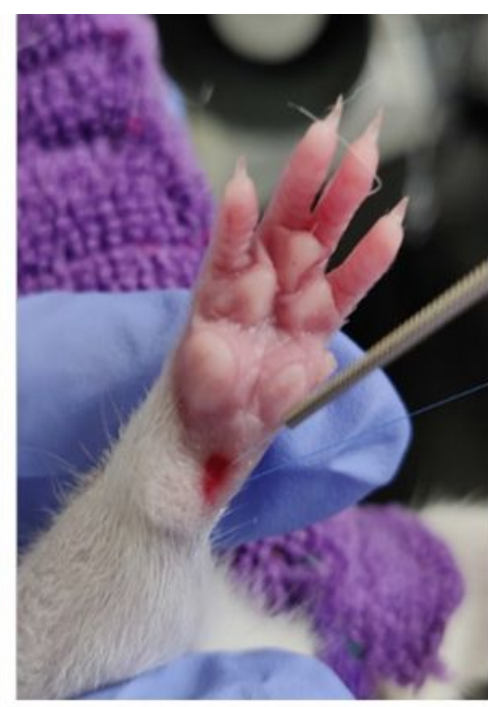

DaLing

Point (PC7)

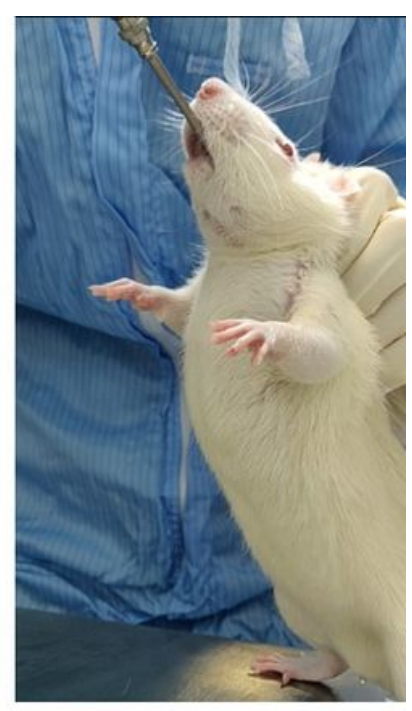

B

\section{Figure 2}

Schematic diagram of acupuncture and fluoxetine operation in rat. A: Flat needling Shangxing(DU23) toward the direction of the nose, the needle was inserted as deep as 3-5mm; Obliquely stab Daling(PC7) toward the direction of Neiguan(PC6), the needle was inserted as deep as 2-3mm; B: Fluoxetine solution $(0.21 \mathrm{mg} / \mathrm{ml})$ by gavage $(1 \mathrm{ml} / 100 \mathrm{~g})$.

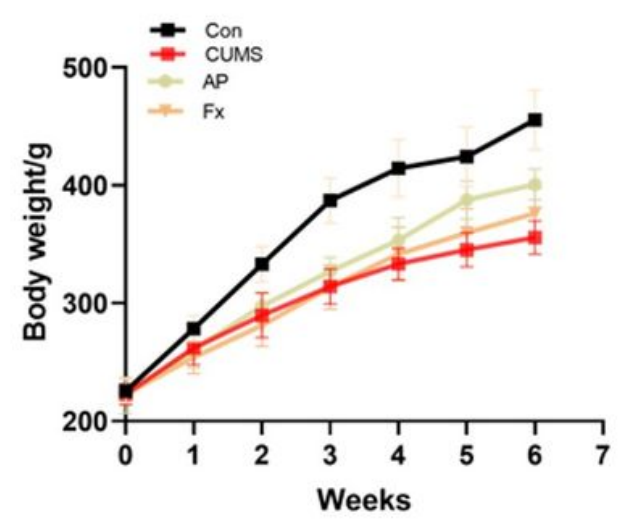

A
Before the experiment

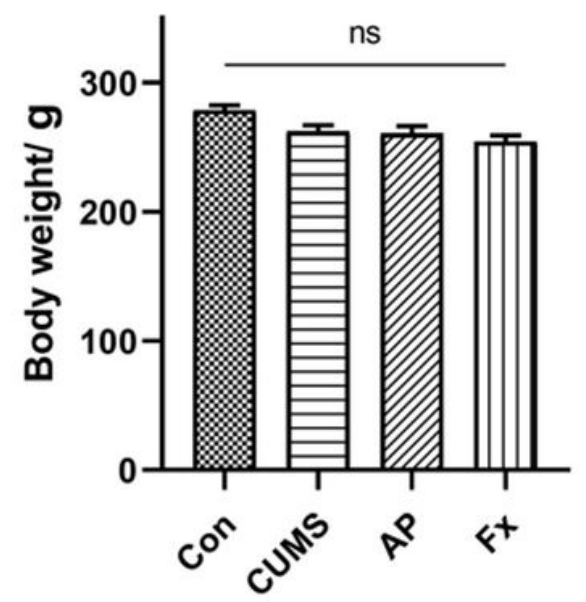

B

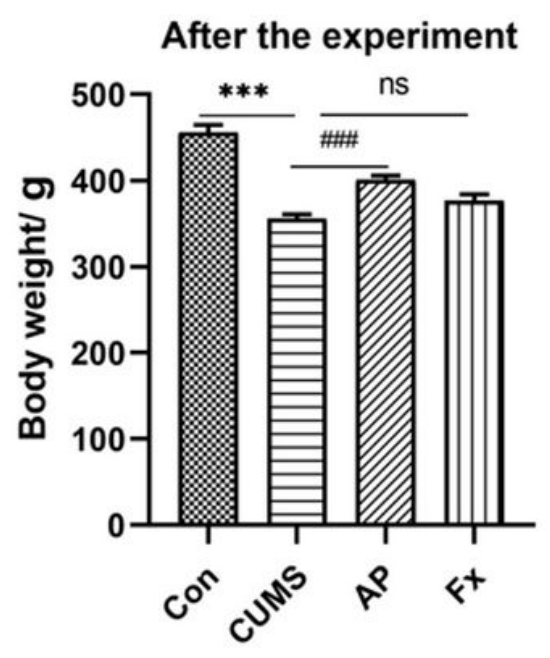

C

\section{Figure 3}

Body weight changes in all groups. A: Comparison of rat's body weight growth trend among four groups during the whole experiment; B: Body weight baseline before the experiment; C: Body weight changes at 
the end of experiment (21day). Compared with the Control group, * $P<0.05$, ** $P<0.01$, *** $P<0.001$; compared with the CUMS group, \# P $<0.05$, \#\# $\mathrm{P}<0.01$, \#\#\# $\mathrm{P}<0.001$, Data represent means \pm SEM.

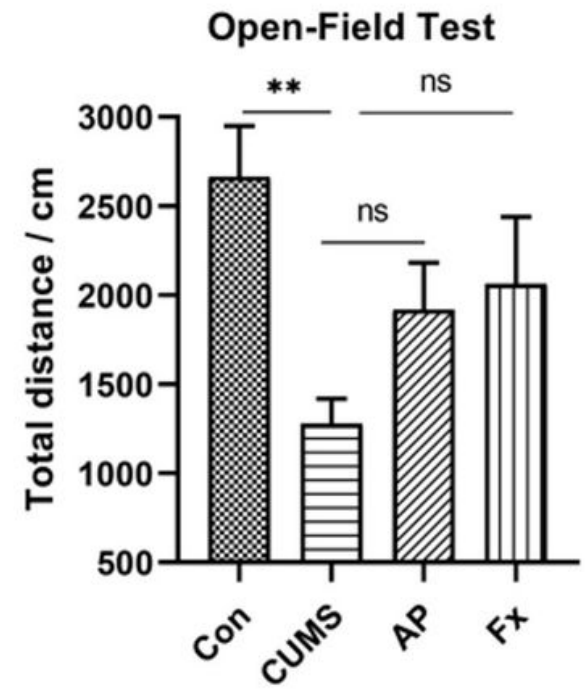

A

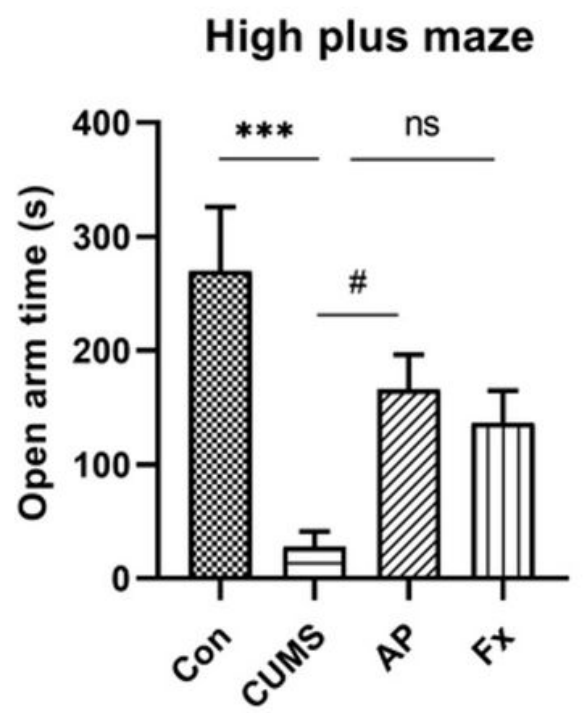

B

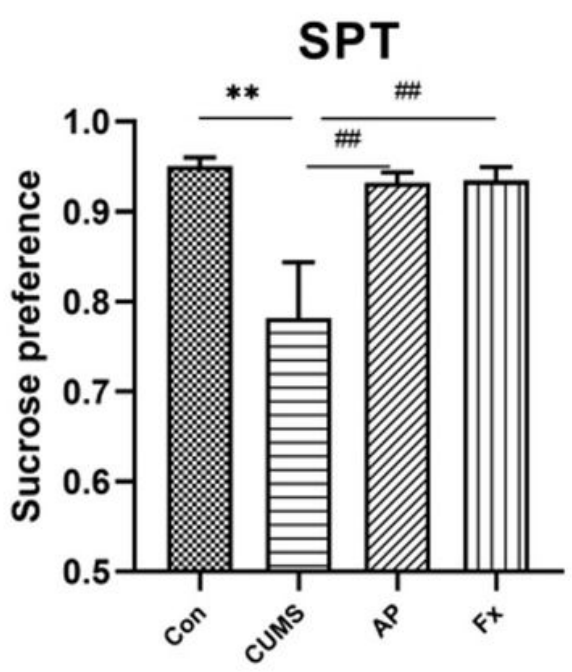

C

Figure 4

Behavior changes in all groups. A: Total distance in the open-field test among four groups; B: Time in open arms of the elevated plus maze among four groups; $\mathrm{C}$ : The percentage of sucrose consumed by the rat at sucrose concentrations of $1 \%$. Compared with the Control group, $P<0.05$, ** $P<0.01$, ${ }^{\star \star \star} P<$ 0.001; compared with the CUMS group, \# P $<0.05$, \#\# P $<0.01$, \#\#\# P $<0.001$, Data represent means \pm SEM.

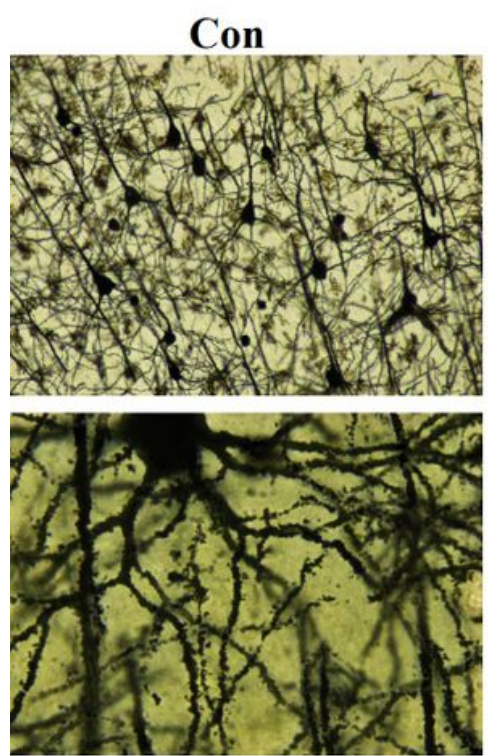

A
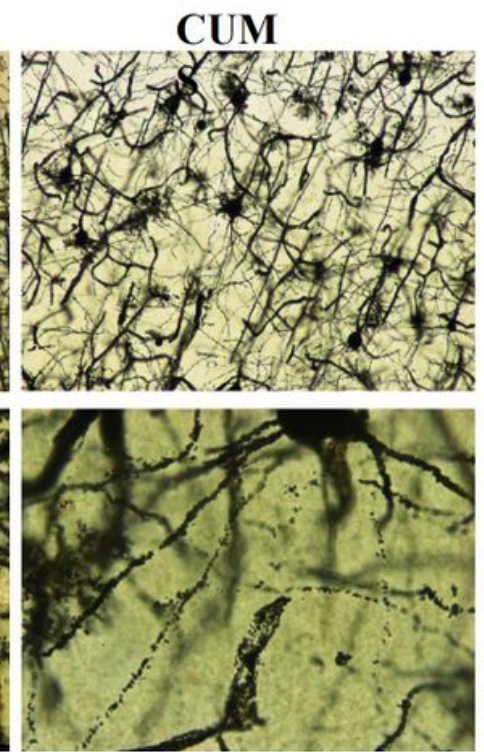

B

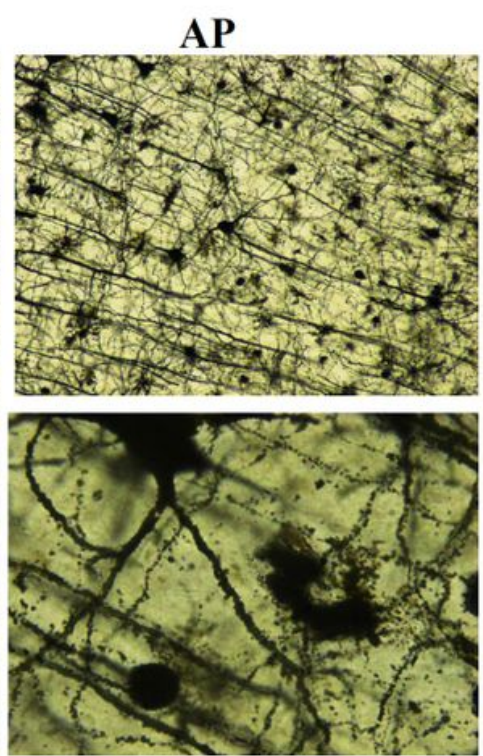

C

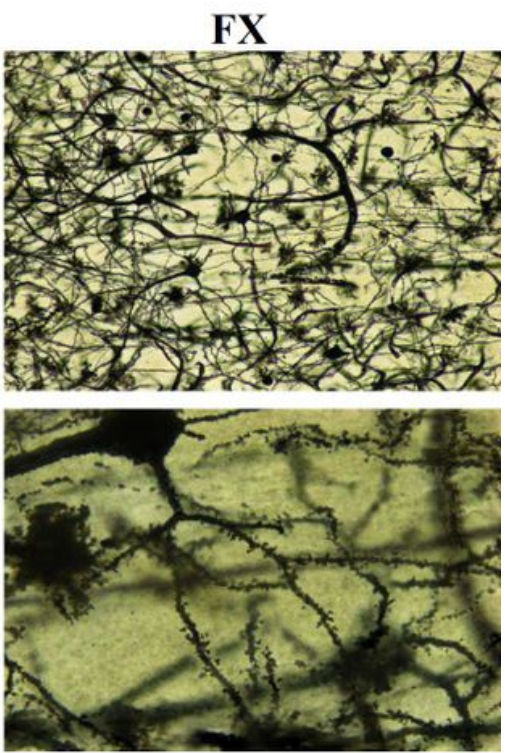

D

Figure 5 
Golgi staining of rat prefrontal cortex [200×(up column) and 1000×(down column)]. A: Control group; B: CUMS group; C: Acupuncture group; D: Fluoxetine group

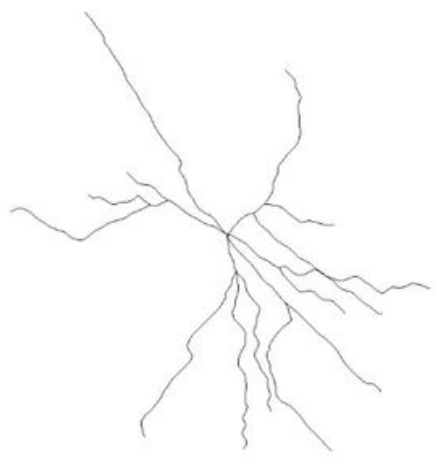

A

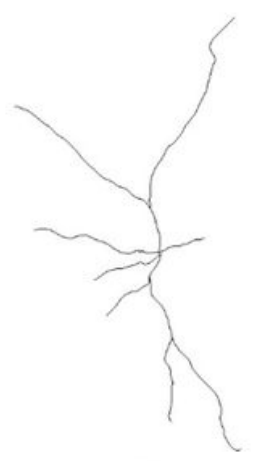

B

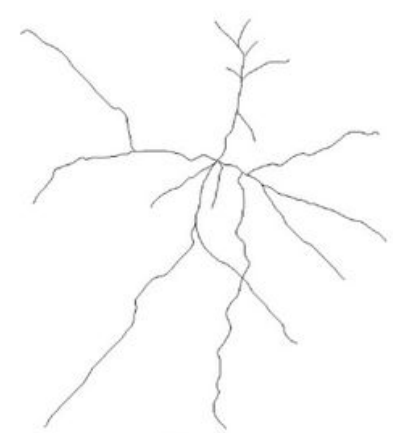

C

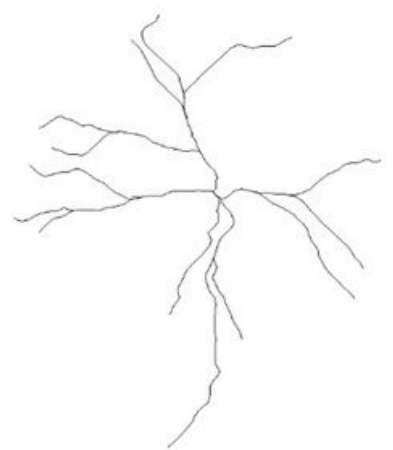

D
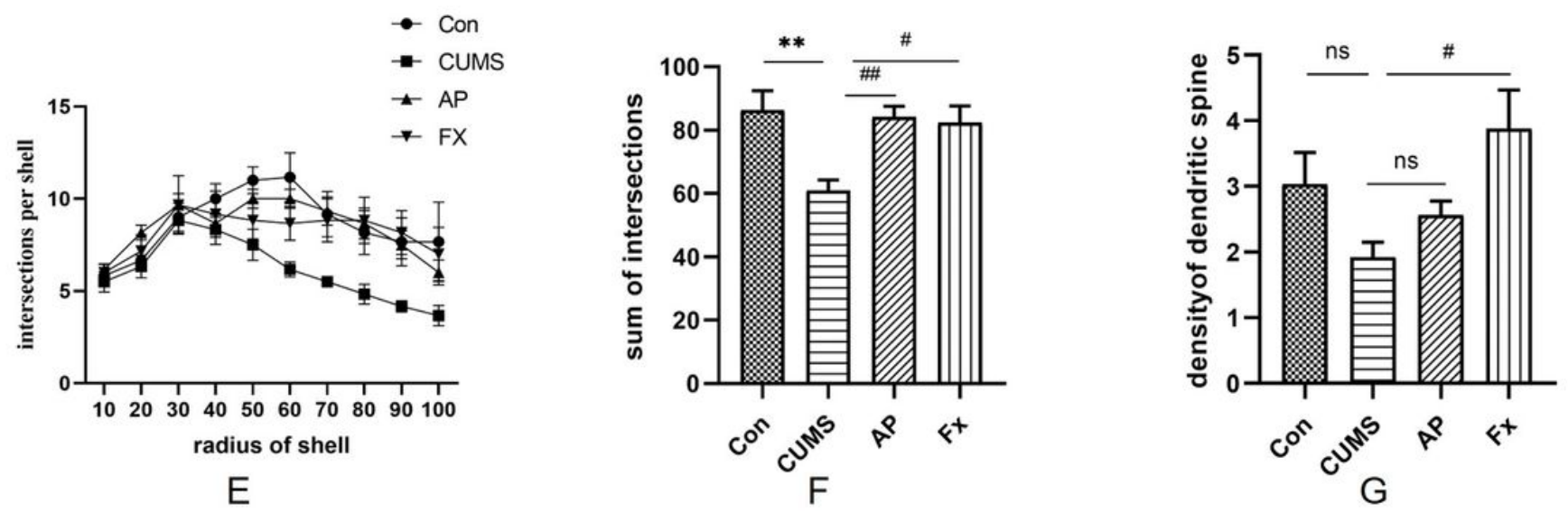

Figure 6

Morphological analyses of dendritic arborization and spine density of prefrontal cortex. A: Control group; B: CUMS group; C: Acupuncture group; D: Fluoxetine group; E: Intersection per shell of pyramidal neuron arborization; F: Sum of intersections of dendrites; G: Density of dendritic spines. Compared with the Control group, ${ }^{\star} \mathrm{P}<0.05$, ${ }^{\star *} \mathrm{P}<0.01$, ${ }^{\star \star *} \mathrm{P}<0.001$; compared with the CUMS group, $\# \mathrm{P}<0.05, \# \# \mathrm{P}<$ 0.01 , \#\#\# $P<0.001$, Data represent means \pm SEM. 

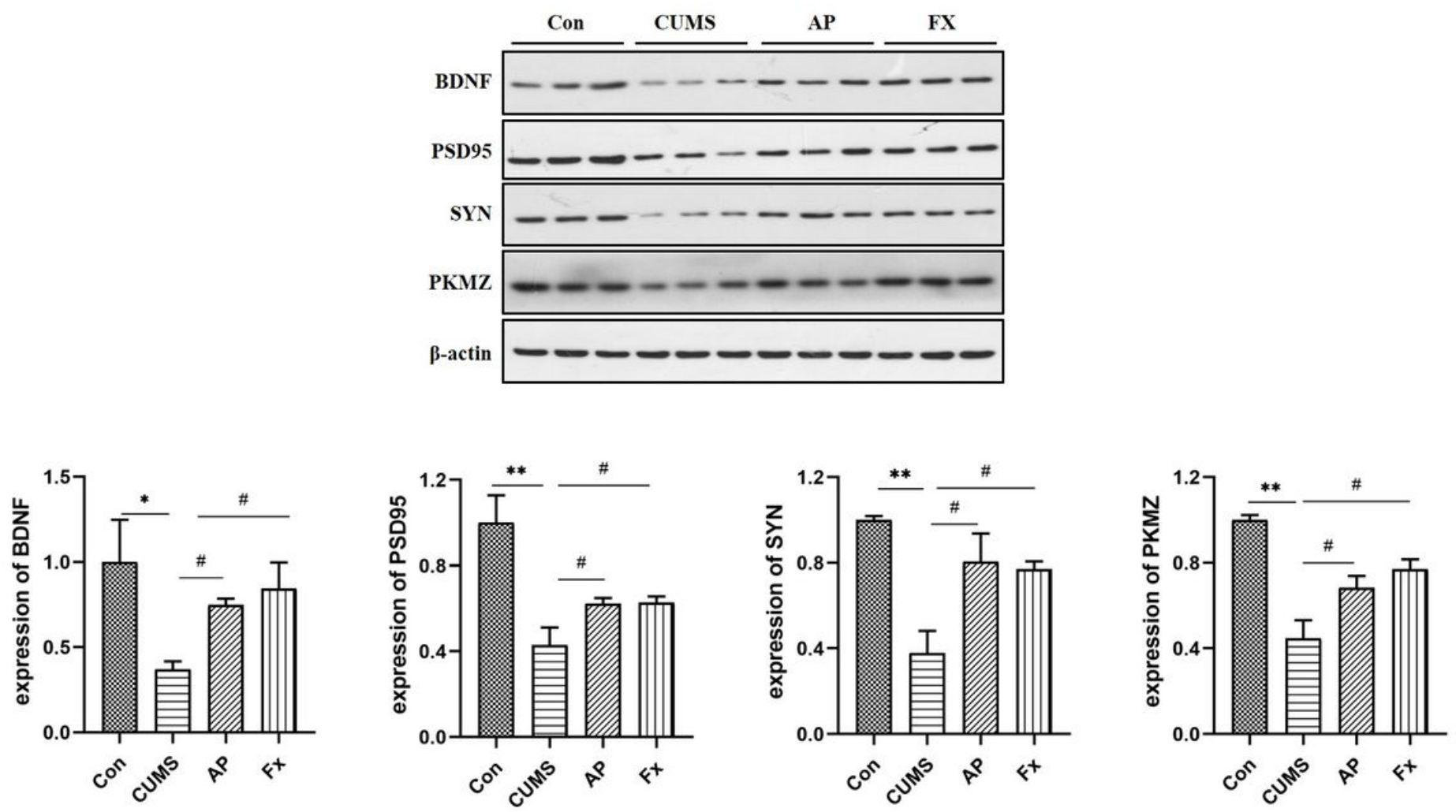

Figure 7

The expression of neural plasticity-related proteins in prefrontal cortex measured by western-blot. A: BDNF; B:PSD95; C: SYN; D: PKMZ. Compared with the Control group, * $P<0.05$, $* \star P<0.01$, ${ }^{\star \star \star} P<0.001$; compared with the CUMS group, \# $P<0.05$, \#\# $P<0.01$, \#\#\# $P<0.001$, Data represent means $\pm S E M$. 

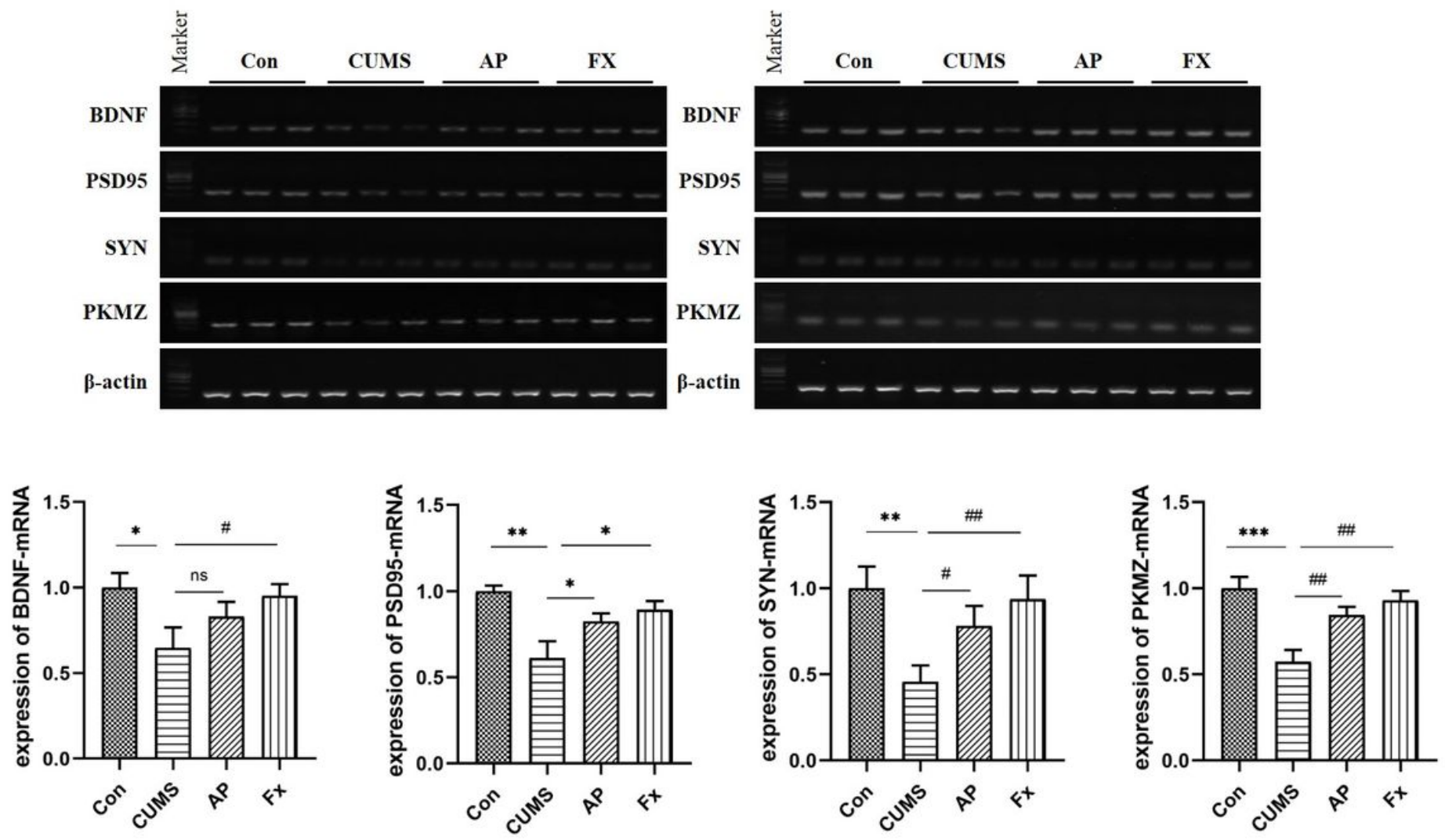

\section{Figure 8}

The mRNA level of neural plasticity-related proteins in prefrontal cortex measured by western-blot. A: BDNF; B:PSD 95; C: SYN; D: PKMZ. Compared with the Control group, * $P<0.05$, ** $P<0.01$, ${ }^{\star \star \star} P<0.001$; compared with the CUMS group, \# $\mathrm{P}<0.05$, \#\# $\mathrm{P}<0.01$, \#\#\# $\mathrm{P}<0.001$, Data represent means $\pm \mathrm{SEM}$. 